\title{
GMR
}

\section{Bos taurus papillomavirus activity in peripheral blood mononuclear cells: demonstrating a productive infection}

\author{
T.C. Melo ${ }^{1,2}$, R.P. Araldi ${ }^{1,3}$, N.S.D. Pessoa ${ }^{1,4}$, P.L. de-Sá-Júnior ${ }^{1}$, R.F. Carvalho1, \\ W. Beçak ${ }^{1}$ and R.C. Stocco ${ }^{1}$ \\ ${ }^{1}$ Laboratório de Genética, Instituto Butantan, São Paulo, SP, Brasil \\ 2Programa de Pós-graduação em Biologia Estrutural e Funcional, \\ Universidade Federal de São Paulo, São Paulo, SP, Brasil \\ ${ }^{3}$ Programa de Pós-graduação Interunidades em Biotecnologia, \\ Instituto de Ciências Biomédicas, Universidade de São Paulo, São Paulo, \\ SP, Brasil \\ ${ }^{4}$ Programa de Pós-graduação em Ciências da Saúde, \\ Faculdade de Ciências Médicas, Universidade Federal de Pernambuco, \\ Recife, SP, Brasil \\ Corresponding author: R.C. Stocco \\ E-mail: rita.stocco@butantan.gov.br \\ Genet. Mol. Res. 14 (4): 16712-16727 (2015) \\ Received August 27, 2015 \\ Accepted November 26, 2015 \\ Published December 11, 2015 \\ DOI http://dx.doi.org/10.4238/2015.December.11.19
}

\begin{abstract}
Bovine papillomavirus (BPV) is an oncogenic virus with mucous and epithelial tropism. Possible productive virus infection in other tissues, such as blood, has been hypothesized. In order to investigate this possibility, three samples of skin papillomas and blood were collected from bovines with BPV infection and five samples of peripheral blood and one sample of normal tissue were collected from a calf without BPV infection. Peripheral blood mononuclear cells (PBMCs) were isolated from whole blood and examined by reverse transcription-polymerase chain reaction, immunofluorescence, in situ hybridization, and electron microscopy. The tissue samples were examined for histopathological and
\end{abstract}


immunohistochemical features. The skin papillomas showed the presence of DNA sequences of BPV-2, BPV-11, and a putative virus type. The blood samples showed DNA sequences of BPV-1, 2, and 4 simultaneously. Immunohistochemistry showed BPV L1 protein in both epithelium and stroma and BPV E2 protein in koilocytes. In situ hybridization confirmed the presence of BPV DNA in PBMCs and immunofluorescence showed nuclear labeling of E2 and L1 BPV proteins in PBMCs. The transcription analysis revealed transcripts of BPV-1 L1, BPV-2 L2, and BPV-4 E7 in blood and papilloma samples of BPV-infected cattle. The comet assay revealed high levels of host cell DNA damage upon BPV infection. Electron microscopy analysis of PBMCs identified the presence of particles in the cytoplasm that are consistent with papillomavirus in size and shape. The productive infection of PBMCs with BPV has been previously discussed and this study provides evidence indicating that PBMCs are a target of BPV.

Key words: BPV; Productive infection; PBMC; Electron microscopy; Comet assay

\section{INTRODUCTION}

Bos taurus papillomavirus (BPV) is an oncogenic virus with mucous and epithelial tropism (White and Howley, 2013), and it is associated with benign lesions (papillomas) that can persist and progress to malignancy, causing devastating economic losses of livestock (Stocco dos Santos et al., 1998). In Brazil, approximately $60 \%$ of cattle are infected with BPV (Stocco dos Santos et al., 1998). The papillomaviruses are classified into 39 genera according to the International Committee on Virus Taxonomy (http://ictvonline.org/virusTaxonomy.asp) and are based on the L1 open reading frame (ORF) nucleotide sequence. Currently, there are 14 types of BPV described in the literature and they are divided into three genera: Deltapapillomavirus (BPV-1, 2, 13 and 14), Epsilonpapillomavirus (BPV-5 and 8), and Xipapillomavirus (BPV-3, 4, 6, 9, 10, 11 and 12). BPV-7 is not included in any genera (Munday et al., 2015). Although considered species-specific, Deltapapillomavirus can infect different animals, including yaks, antelopes, giraffes, zebras, tapirs (Kidney and Berrocal, 2008), buffaloes and horses (Nasir and Reid, 1999). In horses, the infection can produce equine sarcoids (Nasir and Reid, 1999). BPV-1, 2 and 4 are also related to bladder and gastrointestinal cancer (Campo, 1997; Stocco dos Santos et al., 1998).

The BPV genome is divided into three regions: early $(E)$, late $(L)$ and long control region (LCR) (Bocaneti et al., 2014). The E region encodes the E1, E2, E4, E5, E6 and E7 proteins. The L region contains the L1 and L2 ORFs, which encode the capsid proteins and the LCR that contains the origin of replication (ori) (Bocaneti et al., 2014).

The $E 1^{\wedge} E 4-c o n j u g a t e d$ protein represents the most abundant transcript in productive infections and its function has been attributed to cytoskeleton disorganization and virion release (Doorbar, 2013). The E5 oncoprotein is expressed in the basal layer of infected epithelium and is present in the membrane of the endoplasmic reticulum and Golgi complex (Venuti et al., 2011). E5 binds to the platelet-derived growth factor receptor (PDGF $\beta$ ), inducing cell proliferation (Venuti et al., 2011), and promotes the alkalization of the Golgi apparatus membrane, sequestering major histocompatibility complex (MHC)-I and promoting a concomitant reduction in MHC-I and II (Venuti et al., 2011). This process represents an important mechanism of immune evasion. The E6 oncoprotein 
is responsible for the disruption of the actin cytoskeleton and p53 inactivation, which are associated with cell immortalization (White and Howley, 2013). Thus, E6 affects the repair system, leading to the accumulation of DNA lesions (Araldi et al., 2013). E7 promotes the phosphorylation of retinoblastoma protein ( $p R b)$, leading to S phase entry (White and Howley, 2013), and is related to centrosome duplication and numeric aberrations (Duensing et al., 2000). The combined action of oncoproteins E5, E6, and E7 leads to genomic instability and malignancy (Araldi et al., 2013a; Araldi et al., 2015b).

BPV is widespread globally and its dispersion can occur by contact between infected animals, through contact with a virus-contaminated area, such as milking machines, water dispensers, feeders, ropes, and fences, or transmitted by insects (Bocaneti et al., 2014). The infection starts with a microtissue injury, which exposes the peptidoglycan of heparan sulfate in the plasma membrane (McBride et al., 2012). Furthermore, another BPV transmission pathway was described by Stocco dos Santos et al. (1998), who showed the presence of BPV-2 DNA in peripheral blood and reported chromosome instability. The same authors also suggest that the peripheral blood could act as a viral disseminator (Král et al., 2015). Recent studies have reinforced the role of peripheral blood mononuclear cells (PBMCs) as virus carriers, showing that BPV is present in CD4+ and CD8+ lymphocytes (Roperto et al., 2008; Araldi et al., 2014a) and the detection of BPV activity in PBMCs by the comet assay (Araldi et al., 2013a) and flow cytometry (Roperto et al., 2011).

Moreover, the immunodetection of papillomavirus proteins in other tissues, such as placenta, endometrium, chorionic epithelium, umbilical cord (Yaguiu et al., 2008), blastocysts (Calinisan et al., 2002), and semen (Carvalho et al., 2003), also suggests the blood as a vehicle for virus dissemination. This study presents important evidence, in association with previous data (Diniz et al., 2009), suggesting that PBMCs act not only as a viral reservoir but also as a disseminator of BPV to other tissues. Thus, this study demonstrated the productive infection in PBMCs, which was verified by the BPV L1 immunodetection and virus-like particles presence in infected cell which high levels of clastogenesis.

\section{MATERIAL AND METHODS}

\section{Tissue and peripheral blood samples}

Samples were collected from eight animals (B. taurus, Simmental breed): three adult cattle infected with BPV and five healthy calves, which were used as controls. From the infected animals, samples of lesions and blood were obtained. One sample of normal epithelial tissue and five peripheral blood samples from each of the non-infected calves were also collected and used as controls. The farm was selected owing to the absence of the bracken Pteridium aquilinum, which has carcinogenic compounds that can induce DNA damage. The tissues samples were collected by a veterinarian, using local anesthesia ( $2 \%$ lidocaine). The peripheral blood samples were collected using a vacutainer with EDTA (DNA extraction) and heparin (lymphocyte separation). The protocol was approved by the Ethics Committee on Animal Use of Butantan Institute (process No. 1035/13).

\section{DNA extraction}

Peripheral blood samples were subjected to DNA extraction using the QIAamp DNA Mini Kit (Qiagen, Hamburg, Germany) according to the manufacturer instructions. The DNA extraction of cutaneous papilloma samples was performed using the PureLink Genomic DNA Kit (Invitrogen, Carlsbad, CA, USA) according to the manufacturer instructions. The extracted DNA was quantified in 
a spectrophotometer (BioPhotometer Plus; Eppendorf, Hamburg, Germany). The quality of DNA was verified by polymerase chain reaction (PCR) using the primers for bovine $\beta$-globin (Yaguiu et al., 2008).

\section{BPV identification and typing}

Viral identification was performed using the pair of primers FAP59 (5'-TAACWGTIGGICA YCCWTATT-3') and FAP64 (5'-CCWATATCWVHCCATITCICCATC-3'), which were originally described by Forslund et al. (1999). These primers are based on the sequence homology between the L1 ORF of human papillomavirus (HPV) and BPV, which was identified by Ogawa et al. (2004), and they result in an amplicon of $478 \mathrm{bp}$. Samples that did not amplify with the FAP59/FAP64 primer pair were subjected to amplification using specific primers for BPV-1 (F5'- GGAGCGCCTGCTAA CTATAGGA-3', R5'-ATCTGTTGTTTGGGTGGTGAC-3'), which amplifies part of the L1 ORF and results in an amplicon of 301 bp; BPV-2 (F5'-GTTATACCACCCAAAGAAGACCCT-3', R5'-CTGGT TGCAACAGCTCTCTTTCTC-3'), which amplifies the L2 ORF and results in a 164 bp amplicon; and BPV-4 (F5'-GCTGACCTTCCAGTCTTAAT-3', R5'-CAGTTTCAATCTCCTCTTCA-3'), which amplifies the E7 ORF and results in a 170 bp amplicon.

The PCR reactions were performed in a total volume of $50 \mu \mathrm{L}$, employing $200 \mathrm{ng} / \mu \mathrm{L}$ DNA template in a Veriti 96 Well Thermal Cycler (Applied Biosystems, Carlsbad, CA, USA). BPV identification with FAP59/FAP64 primers was done using the following reaction cycle: an initial step of $10 \mathrm{~min}$ at $94^{\circ} \mathrm{C} ; 45$ cycles of $90 \mathrm{~s}$ at $94^{\circ} \mathrm{C}, 90 \mathrm{~s}$ at $50^{\circ} \mathrm{C}$, and $90 \mathrm{~s}$ at $72^{\circ} \mathrm{C}$; and $5 \mathrm{~min}$ at $72^{\circ} \mathrm{C}$. The identification with the specific primers for BPV-1, 2, and 4 was performed using the following reaction cycle: an initial step of $3 \mathrm{~min}$ at $94^{\circ} \mathrm{C} ; 35$ cycles of $50 \mathrm{~s}$ at $94^{\circ} \mathrm{C}, 60 \mathrm{~s}$ at $60^{\circ} \mathrm{C}$, and $60 \mathrm{~s}$ at $72^{\circ} \mathrm{C}$; and $5 \mathrm{~min}$ at $72^{\circ} \mathrm{C}$. For each PCR reaction, negative and positive controls were used. As a positive control, genomes of BPV-1 (AB626707), BPV-2 (M20219.1), and BPV-4 (X05817.1), previously cloned in the bacterial vector pAT153 in Escherichia coli DH5a, were employed.

The resulting PCR products were analyzed on $2 \%$ agarose gel in TAE buffer and stained with $1 \%$ ethidium bromide. The electrophoresis was performed in a Horizon 20:25 gel apparatus (Life Technologies, Carlsbad, CA, USA) at 100 V/400 mA for 120 min. A 100 bp DNA ladder (Invitrogen) was used as a molecular marker. The gel was visualized on a transilluminator MiniBIS Pro (DNR Bio-Imaging Systems, Jerusalem, Israel) and the images were captured by GelCapture version 7.1 software (DNR Bio-Imaging Systems).

The FAP59/FAP64 amplicons were purified using the PureLink Quick Gel Extraction Kit (Invitrogen) according to the manufacturer instructions. For sequencing, $5 \mu \mathrm{L}$ purified amplicons were subjected to sequencing reaction on the ABI PRISM 3730 DNAAnalyzer (Applied Biosystems) using the BigDye Terminator v3.1 Cycle Sequencing Kit (Applied Biosystems) and $2.5 \mu \mathrm{L}$ the respective primers $(5 \mathrm{pM})$. For in silico analysis, the quality of the DNA sequences was assessed and the overlapping fragments were assembled using the software BioEdit version 7.0.9.0 (Ibis Therapeutics, Carlsbad, CA, USA). The sequences were compared using the database of the National Center of Biotechnology (NCBI) with the nucleotide BLAST program (http://blast.ncbi.nlm.gov).

\section{PBMC isolation}

Isolation of PBMCs was performed using a density gradient, employing Ficoll-Paque Plus (GE Healthcare, UK). Peripheral blood $(6 \mathrm{~mL}$ ) was added to Falcon tubes containing $3 \mathrm{~mL}$ FicollPaque Plus and centrifuged for $35 \mathrm{~min}$ at $400 \mathrm{~g}$. The PBMCs were aspirated and $200 \mu \mathrm{L}$ were distributed in slides and $500 \mu \mathrm{L}$ were submitted for cryopreservation at $-80^{\circ} \mathrm{C}$ for mRNA extraction. 


\section{Chromogenic in situ hybridization (CISH) of PBMCs}

$\mathrm{CISH}$ was used to detect BPV genes in blood samples of infected cattle. CISH was performed according to the ZytoFast PLUS CISH Implementation Kit HRP-DAB (Zyto Vision, Germany), using the ZytoFast HPV type 16/18/31/33/35 probe (Zyto Vision). This probe has homology with sequences of L1, E6 and E7 HPV genes. The L1 gene is the most conserved in papillomaviruses (Villiers, 2013) and is used for BPV detection. To control for false positive signals due to nonspecific binding of the anti-digoxigenin antibody or nonspecific precipitation of the chromogen, the DNA probe was not used. To control for nonspecific binding of the DNA probe, reactive probes for other targets contained in the Zyto Vision kit were used. Cells found to be negative for BPV were used as a negative control for other false positive signals. The slides were analyzed using an Axiophot microscope (Carl Zeiss, Hamburg, Germany). The images were captured by the AxioVision software version 4.7.2.

\section{Cryopreservation of PBMCs for transmission electron microscopy}

PBMCs were cryopreserved in $4 \mathrm{~mL}$ RPMI 1640 medium supplemented with $300 \mathrm{mg} /$ $\mathrm{mL}$ L-glutamine, $40 \%$ fetal bovine serum (FBS), and 10\% dimethyl sulfoxide (DMSO). Cells were preserved in sterile $2 \mathrm{~mL}$ cryotubes for $16 \mathrm{~h}$ at $-80^{\circ} \mathrm{C}$ and then transferred to liquid nitrogen where they were stored at $-180^{\circ} \mathrm{C}$ until needed.

PBMCs were defrosted by gradually increasing temperature. For this, cells were removed from the liquid nitrogen and maintained at $-20^{\circ} \mathrm{C}$ for $60 \mathrm{~min}$. Then, the material was maintained at $4^{\circ} \mathrm{C}$ for $30 \mathrm{~min}$ and finally at $37^{\circ} \mathrm{C}$ for $10 \mathrm{~min}$. The PBMCs were centrifuged to remove the cryopreservation media and were suspended in RPMI 1640 medium supplemented with $20 \% \mathrm{FBS}$ at $37^{\circ} \mathrm{C}$. The samples were centrifuged at $600 \mathrm{~g}$ for $5 \mathrm{~min}$. The pellet was fixed in $2 \%$ glutaraldehyde, dehydrated, submitted to negative stain, and embedded in resin. The cells were analyzed using a Leo 906E transmission electronic microscope (Carl Zeiss) and the images were obtained using the Mega View III with the ITEM version E 23082007 software (Olympus Soft Imaging Solutions, Germany), employing a voltaic current of $80 \mathrm{kV}$ and magnification of 60,000 to $120,000 X$ (Araldi et al., 2014b).

\section{mRNA extraction and cDNA construction of blood and tissue samples}

The mRNA was extracted from PBMCs and tissue fragments and preserved in Trizol using chloroform, isopropanol and ethanol. The cDNA was constructed using the ImProm-II Reverse Transcription System (Promega, Madison, USA) and used for transcript analysis by reverse transcription (RT)-PCR of the L1 gene of BPV-1, L2 gene of BPV-2 and E7 gene of BPV-4.

\section{Histopathological analysis of cutaneous papillomas}

A fragment of the representative papilloma skin sample of each animal was used for histopathological analysis. The samples ware fixed in $10 \%$ formalin in PBS, dehydrated in increasing concentrations of ethanol, immersed in paraffin, cut into 5- $\mu \mathrm{m}$ slices, and stained with hematoxylin-eosin (HE). As a negative control, a fragment of skin tissue from an asymptomatic BPV-free calf was also analyzed. 


\section{Immunohistochemical analysis (IHC) of cutaneous papillomas}

For the evaluation of BPV protein expression, samples of cutaneous papillomas were subjected to IHC by using monoclonal antibodies (mAbs) against E2, a protein associated with virus replication and transcription, and the $L 1$ structural protein. The immunodetection of $L 1$ is an important indicator of productive infection, as it is a structural protein associated with the assembly of BPV.

IHC was used to detect BPV protein expression in paraffin-embedded papilloma tissue slices. The technique used the EnVision + system-HRP (AEC) kit (Dako, Carpinteria, CA, USA) with the mAbs against L1 (ab2417, Abcam, USA) and E2 (5H4, Abcam). As controls, a fragment of normal skin (experimental control) and papilloma without primary antibody addition (reaction control) were used. The tissue slices were subjected to deparaffinization and dehydration. After these steps, the slices were treated with 10\% ammonium hydroxide for 10 min and washed in distilled water three times. Antigen retrieval was performed using $0.01 \mathrm{M}$ citrate for 20 min at $95^{\circ} \mathrm{C}$. The slices were washed three times in distilled water and blocked with endogenous peroxidase for 10 min using the peroxidase blocker contained in the kit. The slices were treated with $0.01 \%$ Triton X-100 for $20 \mathrm{~min}$, washed in TBS, and blocked with 5\% BSA for $40 \mathrm{~min}$. Then, the slices were incubated with the primary mAbs in a humid chamber for $16-18 \mathrm{~h}$ at $4^{\circ} \mathrm{C}$. A papilloma sample used as the negative control was incubated with 5\% BSA. After the incubation, the slices were washed with $2 \%$ TBS-Tween (TBS-T) and secondary antibody conjugated to peroxidase was added. The slices were incubated for $1 \mathrm{~h}$ at $4^{\circ} \mathrm{C}$ and washed in $2 \%$ TBS-T three times. The 3-amino-9ethilcarbazole (AEC) chromogenic substrate was applied to the slices for $10 \mathrm{~min}$.

\section{Indirect immunofluorescence (IFI) analysis of PBMCs}

Isolated PBMCs were smeared on slides and fixed in 4\% paraformaldehyde for 30 min at room temperature. The slides were washed in PBS, treated with $0.1 \%$ PBS in Triton X-100 for $15 \mathrm{~min}$, and blocked with $5 \%$ BSA for $40 \mathrm{~min}$ at room temperature. The slides were incubated overnight with the same set of mAbs employed in the IHC analysis using the following dilutions: anti-L1 (1:100) and anti-E2 (1:300). After the incubation, the material was treated with the antimouse secondary antibody conjugated to FITC or TRITC at a 1:250 dilution. The slides were mounted with Prolong Gold (Invitrogen) using nuclear labeling with 4'6'-diamidino-2-phenylindole (DAPI). The slides were analyzed under an Axio Scope A1 fluorescent microscope (Carl Zeiss).

\section{Comet assay of whole blood}

The comet assay was performed according to Araldi et al. (2015a). An aliquot of $10 \mu \mathrm{L}$ $\left(1.0 \times 10^{4}\right.$ cells) whole blood was transferred into $0.2 \mathrm{~mL}$ polypropylene tubes and mixed with 75 $\mu \mathrm{L}$ low melting-point agarose $\left(0.7 \%\right.$ in PBS) at $37^{\circ} \mathrm{C}$. The final volume of $85 \mu \mathrm{L}$ was distributed in pre-coated slides with normal melting-point agarose (1.5\% in PBS). The slides were covered with coverslips and kept at $4^{\circ} \mathrm{C}$ for $20 \mathrm{~min}$. The coverslips were removed and the slides were immersed in lysis solution $(2.5 \mathrm{M} \mathrm{NaCl}, 100 \mathrm{mM}$ EDTA, $10 \mathrm{mM}$ Tris- $\mathrm{HCl}, \mathrm{pH}$ 10, 1\% Triton X-100, 10\% DMSO) for $1 \mathrm{~h}$ at $4^{\circ} \mathrm{C}$. The slides were transferred to a horizontal gel electrophoresis apparatus containing electrophoresis buffer ( $1 \mathrm{mM}$ EDTA, $300 \mathrm{mM} \mathrm{NaOH}, \mathrm{pH}>13$ ) at $4^{\circ} \mathrm{C}$ and kept in this buffer for $40 \mathrm{~min}$ for DNA unwinding. The electrophoresis run was performed for $20 \mathrm{~min}$ at $25 \mathrm{~V}$ $(0.83 \mathrm{~V} / \mathrm{cm})$ and $300 \mathrm{~mA}$. The slides were washed three times in neutralization buffer $(0.4 \mathrm{M}$ Tris$\mathrm{HCl}, \mathrm{pH} 7.5$ ) and fixed in $100 \%$ ethanol. All steps described above were carried out in darkness to 
avoid DNA damage. The slides were stained with $20 \mu \mathrm{L}$ propidium iodide $(4 \mu \mathrm{g} / \mathrm{mL})$ and visualized under an Axio Scope A1 fluorescent microscope (Carl Zeiss). Nucleoids were classified from 0 (without lesions) to 2 (major damage), according to Araldi et al. (2013b), and the number of nucleoids observed per class was multiplied by the class value, resulting in a comet score. The statistical analysis was done by the Mann-Whitney test, with a significance level of $5 \%$. The test was performed using the BioEstat program version 5.3.

\section{RESULTS}

\section{Molecular identification and typing of BPV in tissue and blood samples}

Using the FAP59/FAP64 primers, it was possible to identify the presence of BPV in three tissue samples. The amplicons obtained were sequenced and virus typing showed the presence of BPV-2 in sample 1 (99\% identity), BPV-11 in sample 2 (99\% identity) and a new putative virus type in sample 3 (Figure 1). This new putative virus type was identified as BPV-JN/3SP (GenBank JQ280500.1) and was already described by Melo et al. (2014). However, it was not possible to detect BPV with the FAP59/FAP64 primers in blood samples. PCR with BPV-specific primers showed that BPV-1, -2 and -4 were present in the three papilloma and blood samples (Figure 1 ), indicating virus co-infection in all the samples. The normal skin fragment and blood samples collected from five calves (controls) did not show presence of BPV.

A
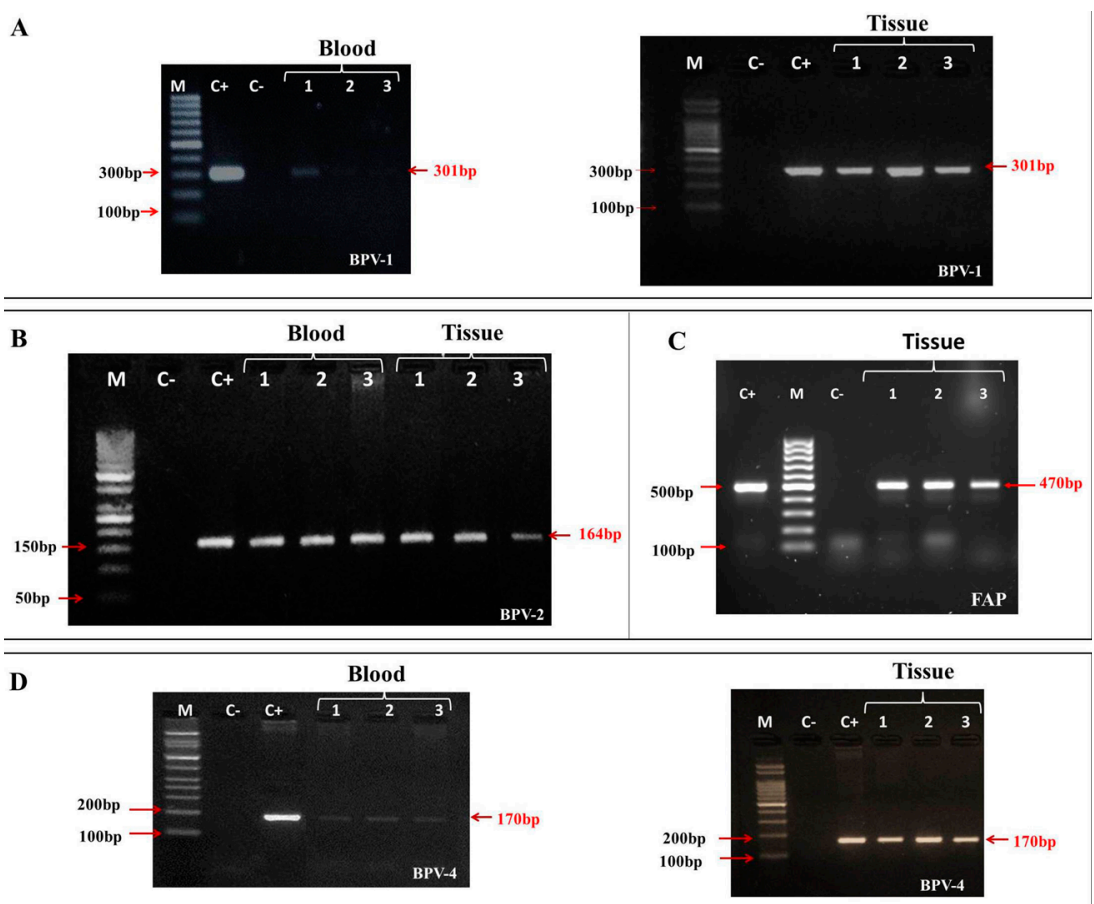

Figure 1. Molecular identification of BPV. (A) Amplified products using specific primers against BPV-1 (A), BPV-2 (B) FAP59/64 (C), or BPV-4 (D) from blood and tissue fragment samples on a $2 \%$ agarose gel stained with ethidium bromide. $\mathrm{M}=100 \mathrm{bp}$ or $50 \mathrm{bp}$ molecular weight ladder; $\mathrm{C}_{+}=$positive control of cloned BPV-1, 2, or 4 genome; $\mathrm{C}_{-}=$negative control; lanes 1, 2, 3 = peripheral blood and tissue samples from BPV-infected animals. Amplicon size is indicated in red. 


\section{Histopathological analysis and BPV protein expression by IHC in papilloma samples}

Histopathological analysis of cutaneous papilloma samples showed irregular keratinization, described as hyperkeratosis, and both ortho- and para-keratotic hyperkeratosis were observed in the three samples. Hypergranulosis and koilocytosis in the granular layer were also observed. Apart from epithelial alterations, all samples showed anaplastic and pleomorphic fibroblasts close to the basal layer (Figure 2). The dermis also revealed a mixed inflammatory infiltrate (Figure 2). Fibroblastic proliferation was observed in samples infected with BPV-2 (Figure 2), which is commonly observed in papillomas infected with Deltapapillomavirus. The normal skin, obtained from an asymptomatic bovine that was PCR-negative for BPV, did not show histopathological alterations.

The results indicate the immunodetection of $L 1$ in both epithelium and dermis in sample 1 (Figure 3). The control reactions did not show labeling for these proteins, confirming the specificity of the procedure. For the E2 protein, immunostaining in the granular layer was observed with nuclear labeling in koilocytes and cytoplasmic labeling in the corneal layer in sample 1 (Figure 3).

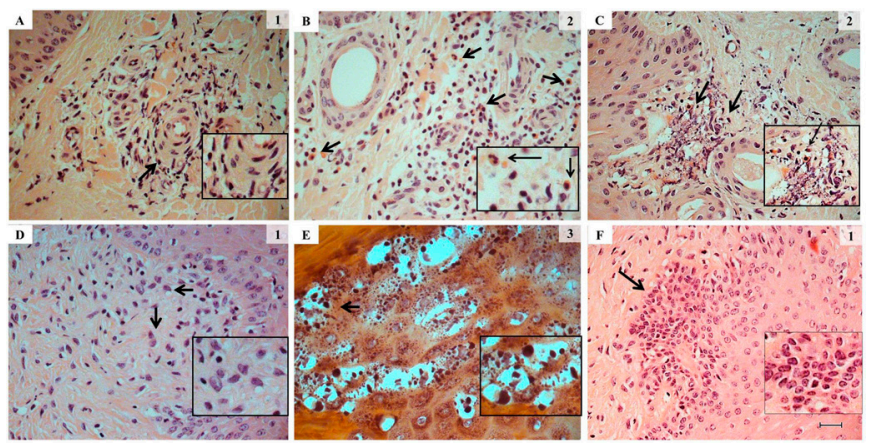

Figure 2. Morphological alteration observed in cutaneous papillomas upon BPV infection. (A) Fibroblastic stroma. (B and C) Anaplastic and pleomorphic fibroblasts and mixed inflammatory infiltrate. (D) Anaplastic and pleomorphic fibroblasts. (E) Koilocytosis and hypergranulosis in granular layer containing keratohyalin granules. (F) Depolarized basal layer. The black arrows indicate the presence of polymorphonuclear cells. Samples 1, 2 and 3 (labeled on the upper right of each panel) were analyzed in total magnification of $400 \mathrm{X} \mathrm{m}$ and the scale bar is $25 \mu \mathrm{m}$.

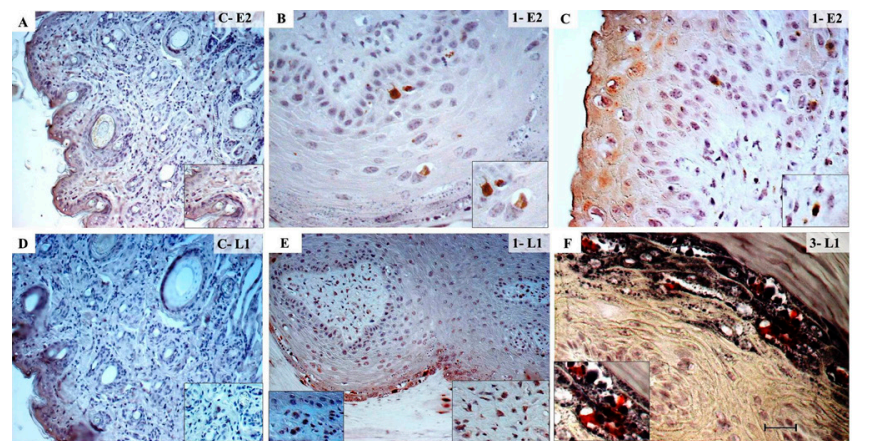

Figure 3. E2 and L1 protein expression in cutaneous papilloma. A) Tissue slice of normal skin (uninfected by BPV) showing absence of E2 protein labelling. Cutaneous papilloma (sample 1) showing E2 labelling in koilocytes in suprabasal layer and in basal cell (B) and, in dermis (C). D) Normal skin showing absence of L1 labelling. Cutaneous papilloma showing L1 labelling in epithelium and dermis of sample $1(\mathbf{E})$ and $3(\mathbf{F})$. Images obtained in total magnification of $100 \mathrm{X}$, with a scale bar of $100 \mu \mathrm{m}(\mathrm{A}$ and $\mathrm{D})$ and, 400X, using a scale bar of $50 \mu \mathrm{m}(\mathrm{B}, \mathrm{C}, \mathrm{E}, \mathrm{F})$. 


\section{CISH of PBMCs}

The presence of BPV sequences in PBMCs was analyzed with $\mathrm{CISH}$ as an additional method to PCR. The results showed the presence of BPV DNA in PBMCs, revealing viral DNA labeling in nuclear, perinuclear, and cytoplasmic regions (Figure 4). Non- infected PBMCs, as well as the control samples (without the probe and with the probe showing no reactivity for BPV), did not reveal any labeling.

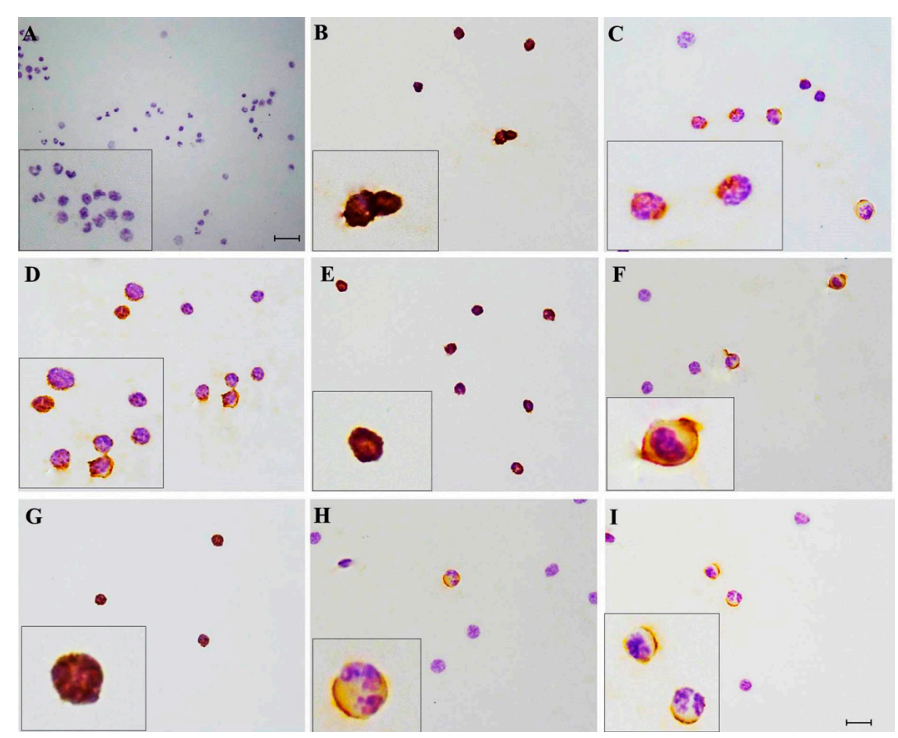

Figure 4. Chromogenic in situ hybridization in PBMCs. (A) PBMCs negative for papillomavirus. (B, E and G) Nuclear labeling in lymphocytes. (D, C, E, H and I) Perinuclear and cytoplasmic labeling in polymorphonuclear cells. (F) Staining in monocyte membranes. Images obtained with 100X objective and scale bar of $6.5 \mu \mathrm{m}$.

\section{BPV gene expression in PBMC samples infected with BPV}

PBMCs positive for BPV sequences were subjected to RT-PCR and immunofluorescence analysis to confirm viral expression. Analysis by RT-PCR showed transcription of L1 of BPV-1, L2 of BPV-2 and E7 of BPV-4 in both blood and papilloma tissue (Figure 5). The immunofluorescence analysis showed E2 protein expression in the nuclear region as well as expression of the structural L1 protein in PBMCs (Figure 6). The immunodetection of these proteins was observed in the three blood samples collected from animals positive for BPV, confirming the expression of BPV proteins in PBMCs from all analyzed samples.

\section{Analysis of BPV particles in PBMCs by transmission electron microscopy}

Considering that BPV sequences were detected by PCR and CISH in PBMCs, we verified the presence of BPV particles in PBMCs using electron microscopy. The electron microscopy showed the presence of particles in the cytoplasm of PBMCs that are similar to BPV particles in shape and diameter (between 45-60 nm) according to the literature (Araldi et al. 2014b) (Figure 7). 


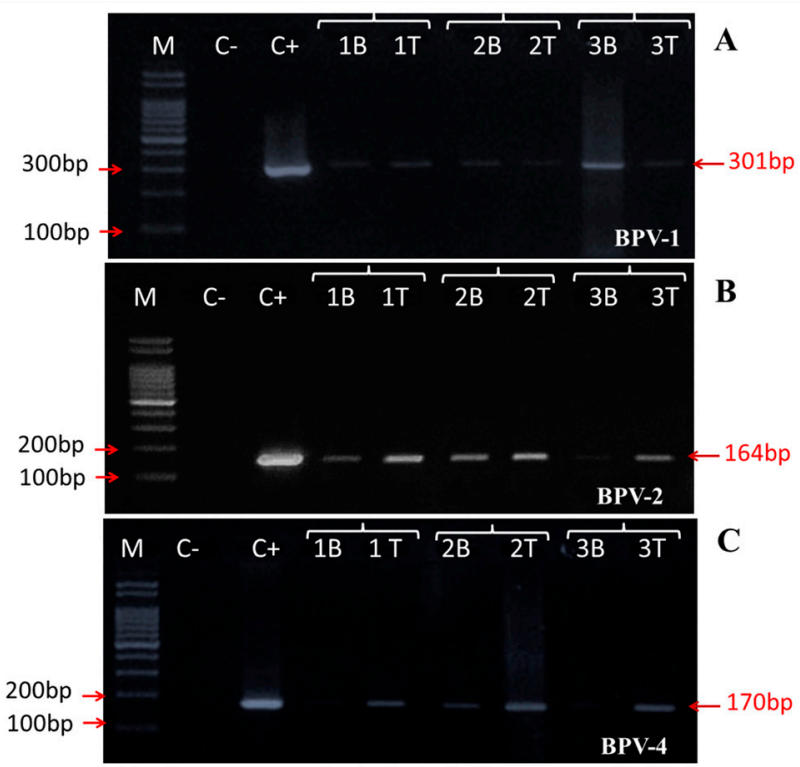

Figure 5. Transcript products of L1, L2, and E7 of BPV in cattle blood and tissue. RT-PCR showing BPV-1 L1 (A), BPV-2 L2 (B), and BPV-4 E7 (C) expression on a $2 \%$ agarose gel stained with ethidium bromide. $M=100$ bp molecular weight ladder; $\mathrm{C}^{+}=$positive control; $\mathrm{C}-=$ negative control; blood $(1 \mathrm{~B}, 2 \mathrm{~B}$ and $3 \mathrm{~B})$ and tissue $(1 \mathrm{~T}, 2 \mathrm{~T}$ and $3 \mathrm{~T})$ samples. Amplicon size is indicated in red.

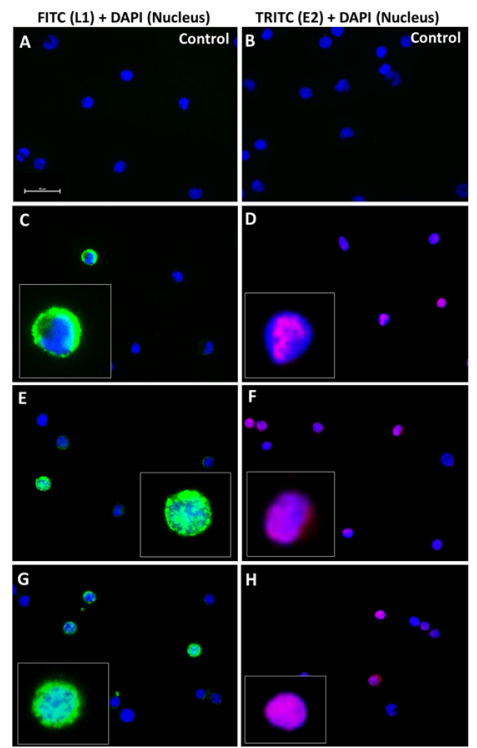

Figure 6. E2 and L1 protein expression in BPV-infected PBMCs. (A and B) Uninfected and unlabeled blood. (C and D) PBMCs from sample 1 with L1 and E2 nuclear labeling. (E and F) PBMCs from sample 2 with L1 and E2 cytoplasmic and nuclear labeling. ( $\mathbf{G}$ and $\mathbf{H}$ ) PBMCs from sample 3 with L1 cytoplasmic and nuclear labeling and E2 nuclear labeling. FITC (L1) stains cells green and TRITC (E2) stains cells red. Nuclei are labeled with DAPI in blue. Images were obtained with 100X objective and a scale bar of $50 \mu \mathrm{m}$ is shown. 

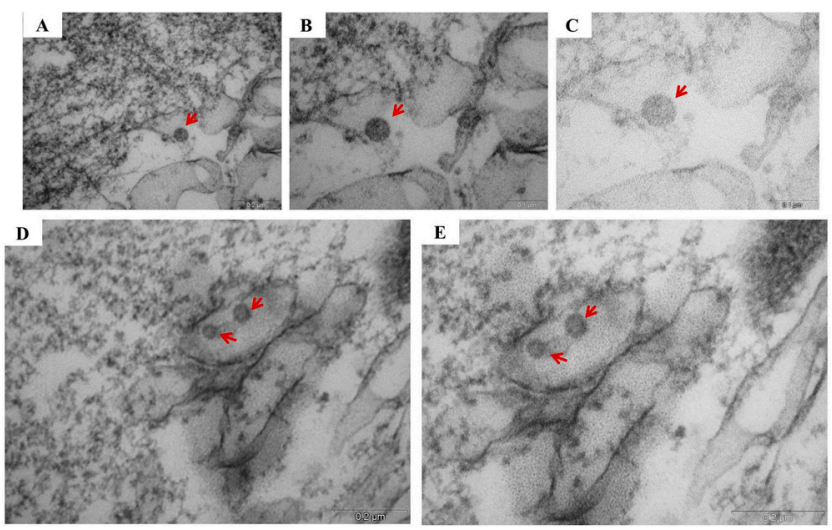

Figure 7. Identification of BPV viral particles in PBMCs with electron microscopy. (A-E) Dense particles are seen in the cytoplasm. Particles 45-55 nm in diameter are identified as BPV-like particles (red arrows) in PBMCs. (A) 60,000X with a scale bar of $0.2 \mu \mathrm{m}$; (B) 100,000X with a scale bar of $0.1 \mu \mathrm{m}$; (C) $129,300 \mathrm{X}$ with a scale bar of $0.1 \mu \mathrm{m}$; (D) $60,000 \mathrm{X}$ with a scale bar of $0.2 \mu \mathrm{m}$; and (E) $77,500 \mathrm{X}$ with a scale bar of $0.2 \mu \mathrm{m}$.

\section{Clastogenic analysis of PBMCs infected with BPV by comet assay}

The comet assay analysis results are presented in Figure 8. The statistical comparison between BPV-infected and non-infected blood samples showed different clastogenic levels. The Mann-Whitney test revealed statistical differences $[U=0.00, Z(U)=2.4492, P=0.0072]$. A high frequency of nucleoids class 2 was observed, indicating the maximum DNA damage in samples infected with BPV (Figure 8).

A

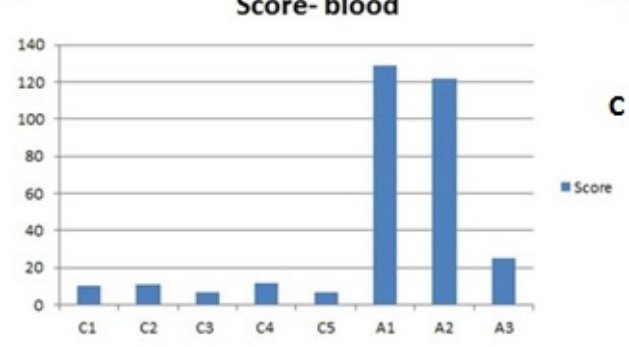

B

C
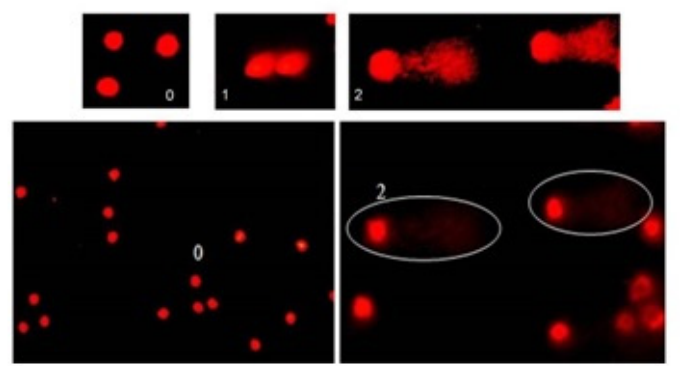

Figure 8. Clastogenic levels observed by comet assay in BPV-infected samples. A) Graphical representation of scores obtained (Y axis): BPV-free (C1-C5) and BPV-infected (A1-A3) samples. The Mann-Whitney test revealed statistical differences $(U=0.00, Z(U)=2.4492, P=0.0072)$ between infected and uninfected bovine samples. (B) Class of nucleoids: 0 - without DNA damage, 1 - intermediate DNA damage level and 2 - maximum DNA damage level. C) Nucleoids class 0, observed in BPV-free samples and class 2, verified in BPV infected sample.

\section{DISCUSSION}

The histopathological analysis of the cutaneous papillomas showed tissue alterations in both epithelium and stroma. The alterations observed in the epithelium resulted from the synergistic action of the E5, E6 and E7 oncoproteins (Araldi et al., 2015b). These proteins induce S phase entry, leading to a mitogenic stimulus, which results in the observed acanthosis and parakeratosis. 
The koilocytosis is a result of virus assembly, considered by some as a cytopathic effect frequently observed in papilloma samples (Carvalho et al., 2013). However, the dermis alterations are rarely reported in the literature. One reason for this could be the few studies involving histopathological analysis of papillomas and the fact that the papillomaviruses exhibit tropism for epithelial and mucosal tissue. The presence of anaplastic and pleomorphic fibroblasts was already reported in fibropapillomas (Jelínek and Tachezy, 2005) and BPV-infected equine sarcoids (Yuan et al., 2010). Moreover, it is known that BPV can infect fibroblasts (Yuan et al., 2010) and fibroblastic transformation has been correlated with possible E5 diffusion into the dermis and E7 oncoprotein action (Nicholls et al., 2001). Although PCR identified the presence of BPV sequences in papilloma samples, the lesion fragments were subjected to IHC to analyze viral protein expression in infected stroma.

According to Doorbar (2013), BPV protein expression is regulated spatially and temporally by epithelial differentiation. Early proteins are expressed in the undifferentiated layers of the epithelium, whereas the late proteins are expressed in the differentiated layers, where virus assembly occurs (Venuti et al., 2011). However, the IHC results showed E2 protein expression in the corneal layer and unexpected L1 protein expression in less differentiated layers, including the basal cells and stroma. These results lead to discussions about the temporal expression of the BPV proteins. This phenomenon was also observed by Nicholls et al. (2001), who found the E2 and E7 proteins in the nuclei of mature koilocytes of papillomas infected with canine oral papillomavirus (COPV). Similar results were also described by Coupe et al. (2012), who observed the presence of E1 and E2 transcripts in the nuclei of cells in the suprabasal layers of neoplastic lesions infected with HPV. The authors also noted the absence of E1 and E2 transcripts in the basal layer.

This study observed the immunodetection of the L1 structural protein in the stroma, which has already been described (Araldi et al., 2015). According to the literature, productive infection is characterized by the expression of late proteins, such as L1 (Nasir and Reid, 1999; Roperto et al., 2011). Thus, the immunodetection of $L 1$ suggests a productive infection of the dermis, where anaplastic and pleomorphic fibroblasts were observed in the dermal-epidermal junction. However, these clues into the transformation of fibroblasts are a result of the synergistic actions of the E5, E6 and E7 oncoproteins (DeMasi et al., 2007). Although preliminary data, it is suggested that papillomaviruses in the stroma can migrate to peripheral blood, establishing a latent infection (Araldi et al., 2015b).

Since 1998, the role of peripheral blood as an agent of virus dissemination to other tissues has been discussed (Stocco dos Santos et al., 1998). The presence of BPV sequences in peripheral blood has already been described in the literature (Stocco dos Santos et al., 1998; Roperto et al., 2008; Diniz et al., 2009; Melo et al., 2011; Araldi et al., 2013b). The peripheral blood does not represent the first site of virus contact, according to the infection pathway commonly described (Florin et al., 2012). These data suggest the blood acts as a virus carrier to internal organs, as BPV has been associated with bladder and gastrointestinal cancers (Campo, 1997). Moreover, sequences of BPV were detected in other sites, such as urine, breast milk, semen, oocytes, spermatozoids (Carvalho et al., 2003) and placenta (Roperto et al., 2012). The presence of papillomavirus in the reproductive tract is not seen exclusively with BPV infection, but is also observed with HPV (Calinisan et al., 2002). HPV activity in blastocysts and trophoblasts was verified as well as DNA damage related to virus oncoprotein action (Calinisan et al., 2002; Grce et al., 2012). Although the vertical transmission of BPV was proposed in 1998 (Stocco dos Santos et al., 1998), productive infection in placenta was observed in 2012, when the immunodetection of the E2, E5 and L1 proteins of BPV-2 were detected in the chorionic epithelium (Roperto et al., 2012). The productive infection was demonstrated by the presence of viral particles in the placenta epithelium, which were observed by electron microscopy (Roperto et al., 2012). 
Although current studies have shown the expression of E5 and L1 of BPV-1 and -2 in CD4+ and CD8+ cells (Roperto et al. 2008, 2011), as well as BPV activity in PBMCs by comet assay in both symptomatic and asymptomatic cattle (Araldi et al., 2013a), the presence of BPV virions in PBMCs have not been identified. In this study, PCR results showed the co-infection of BPV-1, -2 and -4 in PBMCs, which has already been described in peripheral blood (Carvalho et al., 2013; Araldi et al., 2014a). These BPV types are the most frequently identified and are associated with bladder and gastrointestinal cancers (Campo, 1997). In this study, the BPV sequences in PBMCs were confirmed by $\mathrm{CISH}$, which showed a pattern of labeling compatible with the episomal state, as proposed by Foresta et al. (2013). The IFI results allowed the identification of E2 and L1 expression in PBMCs, which was associated with the presence of L1, L2 and E7 virus transcripts and suggest the presence of BPV activity in PBMCs. The immunodetection of E2 indicates that BPV can replicate in blood cells (Liu et al., 2010) since the E2 protein is involved in replication, transcription, and episome maintenance. The major evidence for productive infection in PBMCs comes from the detection of L1 protein expression, as it is the major capsid protein (Campo, 1997). Accordingly, its expression has already been shown in placenta tissue, in which the productive infection of BPV has been observed (Roperto et al., 2012). Consequently, the peripheral blood does not only act as a site of viral latency, but also as a possible vehicle for dissemination of BPV particles. Moreover, studies have shown that the papillomaviruses are able to evade the immune system, causing a reduction in $\mathrm{MHC}-\mathrm{I}$ and $\mathrm{MHC}-\mathrm{Il}$ expression and reducing virus recognition by CD8+ and CD4+ cells, respectively (Bolpetti et al., 2010). Thus, the virus also reduces the expression of the Toll-like 9 receptor (Bolpetti et al., 2010).

Currently, the productive infection of BPV in PBMCs has been discussed but not proven. Although this work has shown the presence of BPV DNA sequences by PCR and CISH and the expression of early and late proteins by IFI and RT-PCR in PBMCs, the major evidence for the presence of viral particles in the blood was obtained with electron microscopy, which showed the presence of BPV particles in the cytoplasm of PBMCs. The identification of viral particles by electron microscopy is required to prove the productive infection. Altogether, this work strongly suggests that the peripheral blood acts as both a BPV carrier and a productive infection site. These data indicate a new virus infection pathway already proposed by Stocco dos Santos et al. (1998) and Araldi et al. (2013a), in which the infiltrated leukocytes can carry BPV particles to a site exposed to a micro-injury. This model also explains the frequent recurrence of papillomatosis observed in cattle and possibly in equines affected by BPV-induced sarcoids. The comet assay showed a high clastogenic level in PBMCs infected with BPV. This result reinforces the point that DNA breaks result from viral activity, leading to genomic instability and eventual malignancy (Stocco dos Santos et al., 1998; Melo et al., 2011; Araldi et al., 2013a). The clastogenic action of HPV has already been reported and is associated with spontaneous abortions (Calinisan et al., 2002). As BPV infection in bovines is considered a good model for HPV studies, the possible productive infection in the blood should be better investigated, as some evidence suggests that HPV can also establish a productive infection (Foresta et al., 2013).

In summary, this study provides further evidence that PBMCs can support a productive infection of BPV, leading to the hypothesis that the blood acts not only as a latency site but also as a carrier and disseminator of virus. The blood cells can transport the virus to other tissues, explaining the presence of bladder and gastrointestinal cancer in bovines not exposed to injuries but infected with BPV. 


\section{Conflicts of interest}

The authors declare no conflict of interest.

\section{ACKNOWLEDGMENTS}

The authors thank Magna Soares for academic support; Ministério de Ciência, Tecnologia e Inovação/Conselho Nacional de Desenvolvimento Científico e Tecnológico (CNPq Proc. \#402539/2011-7) and Institute Butantan for financial support; Coordenação de Aperfeiçoamento Pessoal de Nível Superior (CAPES) and CNPq for scholarships for postgraduate students; and Martin W. Breuer for all efforts and structural support.

\section{REFERENCES}

Araldi R, Melo T, Diniz N, Carvalho R, et al. (2013a). Bovine papillomavirus clastogenic effect analyzed in comet assay. Bio. Res. Int. 2013: 1-7.

Araldi R, Oliveira D, Silva D, Mendes T, et al. (2013b). Análise do potencial mutagênico dos esteróides anabólicos androgênicos (EAA) e da L-Carnitina mediatnte o teste do micronúcleo em eritrócitos policromáticos. Rev. Bras. Med. do Esporte. 19: 448-51.

Araldi R, Carvalho R, Melo T, Diniz N, et al. (2014a). Bovine papillomavirus in beef cattle: first description of BPV-12 and putative type BAPV8 in Brazil. Genet. Mol. Res. 13: 5644-53.

Araldi R, Giovanni D, Melo T, Diniz N, et al. (2014b). Bovine papillomavirus isolation by ultracentrifugation. J. Virol. Methods. 208: 119-24.

Araldi R, de Melo T, Mendes T, De-Sá-Júnior P, et al. (2015a). Using the comet and micronucleus assays for genotoxicity studies: A review. Bio. Pharma. 72: 74-82.

Araldi R, Melo T, Neves A, Spadacci-Morena D, et al. (2015b). Hyperproliferative action of bovine papillomavirus (BPV): Genetics and histopathological aspects. Genet. Mol. Res. in press. 14: 12942-54.

Bocaneti F, Altamura G, Corteggio A, Velescu E, et al. (2014). Bovine papillomavirus: new insights into an old disease. Transbound Emerg. Dis. 1-10.

Bolpetti A, Silva JS, Villa LL, Lepique AP (2010). Interleukin-10 production by tumor infiltrating macrophages plays a role in Human Papillomavirus 16 tumor growth. BMC Imunol. 11: 27.

Calinisan J, Chan S, King A, Chan P (2002). Human papillomavirus and blastocyst apoptosis. J. Assist. Reprod. Genet. 19: $132-6$.

Campo M (1997). Bovine papillomavirus and cancer. Vet. J. 154: 175-88.

Carvalho C, Freitas A, Brunner O, Góes L, et al. (2003). Bovine papillomavirus type 2 in reproductive tract and gametes of slaughtered bovine females. Brazilian J. Microbiol. 34 (Suppl.1): 82-4.

Carvalho R, Sakata S, Giovanni D, Mori E, et al. (2013). Bovine papillomavirus in Brazil: detection of coinfection of unusual types by a PCR-RFLP method. Biomed Res. Int. 2013: 270898.

Coupe VM, González-Barreiro L, Gutiérrez-Berzal J, Melián-Bóveda A, et al. (2012). Transcriptional analysis of human papillomavirus type 16 in histological sections of cervical dysplasia by in situ hybridisation. J. Clin. Pathol. 65: 164-70.

DeMasi J, Chao M, Kumar A, Howley P. (2007). Bovine papillomavirus E7 oncoprotein inhibits anoikis. J. Virol. 81: 9419-25.

Diniz N, Melo T, Santos J, Mori E, et al. (2009). Simultaneous presence of bovine papillomavirus in blood and in short-term lymphocyte cultures from dairy cattle in Pernambuco, Brazil. Genet. Mol. Res. 8: 1474-80.

Doorbar J. (2013). The E4 protein; structure, function and patterns of expression. Virology. 445: 80-98.

Duensing S, Lee L, Duensing A, Basile J, et al. (2000). The human papillomavirus type 16 E6 and E7 oncoproteins cooperate to induce mitotic defects and genomic instability by uncoupling centrosome uplication from the cell division cycle. Proc. Natl. Acad. Sci. U.S.A. 97: 10002-7.

Florin L, Sapp M and Spoden G. (2012). Host-cell factors involved in papillomavirus entry. Med. Microbiol Immunol. 201: 437-48.

Foresta C, Bertoldo A, Garolla A, Pizzol D, et al. (2013). Human papillomavirus proteins are found in peripheral blood and semen Cd20+ and Cd56+ cells during HPV-16 semen infection. BMC ilnfectipus Dis. 13: 593.

Forslund O, Antonsson A, Nordin P, Stenquist B, et al. (1999). A broad range of human papillomavirus types detected with a general PCR method suitable for analysis of cutaneous tumours and normal skin. J. Gen. Virol. 80: 2437-43.

Jelínek F and Tachezy R. (2005). Cutaneous papillomatosis in cattle. J. Comp. Pathol. 132: 70-81.

Kidney B and Berrocal A. (2008). Sarcoids in two captive tapirs (Tapirus bairdii): clinical, pathological and molecular study. Vet. Dermatol. 19: 380-4. 
Král J, Band’ouchová H, Brichta J, Kováčová V, et al. (2015). Papillomavirus infection of roe deer in the Czech Republic and fibropapilloma-associated levels of metallothionein, zinc, and oxidative stress. Acta Vet. Brno. 84: 105-11.

Liu X, Schuck S and Stenlund A. (2010). Structure-based mutational analysis of the bovine papillomavirus E1 helicase domain identifies residues involved in the nonspecific DNA binding activity required for double trimer formation. J. Virol. 84: 4264-76.

McBride A, Sakakibara N, Stepp W and Jang M. (2012). Hitchhiking on host chromatin: how papillomaviruses persist. Biochim. Biophys Acta. 1819: 820-5.

Melo T, Carvalho R, Mazzucchelli-de-Souza J, Diniz N, et al. (2014). Phylogenetic classification and clinical aspects of a new putative Deltapapillomavirus associated with skin lesions in cattle. Genet. Mol. Res. 13: 2458-69.

Melo TC, Diniz N, Campos SRC, Ferraz OP, et al. (2011). Cytogenetic studies in peripheral blood of bovines afflicted by papillomatosis. Vet. Comp. Oncol. 9: 269-74.

Munday J, Thomson N, Dunowska M, Knight C, et al. (2015). Genomic characterisation of the feline sarcoid-associated papillomavirus and proposed classification as Bos taurus papillomavirus type 14. Vet. Microbiol. 177: 289-95.

Nasir L and Reid S. (1999). Bovine papillomaviral gene expression in equine sarcoid tumours. Virus Res. 61: 171-5.

Nicholls P, Doorbar J, Moore R, Peh W, et al. (2001). Detection of viral DNA and E4 protein in basal keratinocytes of experimental canine oral papillomavirus lesions. Virology [Internet]. 284: 82-98.

Ogawa T, Tomita Y, Okada M, Shinozaki K, et al. (2004). Broad-spectrum detection of papillomaviruses in bovine teat papillomas and healthy teat skin. J. Gen. Virol. 85: 2191-7.

Roperto S, Brun R, Paolini F, Urraro C, et al. (2008). Detection of bovine papillomavirus type 2 in the peripheral blood of cattle with urinary bladder tumours: possible biological role. J. Gen. Virol. 89: 3027-33.

Roperto S, Comazzi S, Ciusani E, Paolini F, et al. (2011). PBMCs are additional sites of productive infection of bovine papillomavirus type 2. J. Gen. Virol. 92: 1787-94.

Roperto S, Borzacchiello G, Esposito I, Riccardi M, et al. (2012). Productive infection of bovine papillomavirus type 2 in the placenta of pregnant cows affected with urinary bladder tumors. PLoS One. 7: 1-9.

Stocco dos Santos RC, Lindsey CJ, Ferraz OP, Pinto JR, et al. (1998). Bovine papillomavirus transmission and chromosomal aberrations: an experimental model. J. Gen. Virol. 79: 2127-35.

Venuti A, Paolini F, Nasir L, Corteggio A, et al. (2011). Papillomavirus E5: the smallest oncoprotein with many functions. Mol. Cancer. 10: 140.

Villiers E. (2013). Cross-roads in the classification of papillomaviruses. Virology 445: 2-10.

White E and Howley P. (2013). Proteomic approaches to the study of papillomavirus - host interactions. Virology 435: 57-69.

Yaguiu A, Dagli M, Birgel-Júnior E, Reis B, et al. (2008). Simultaneous presence of bovine papillomavirus and bovine leukemia virus in different bovine tissues: in situ hybridization and cytogenetic analysis. Genet. Mol. Res. 7: 487-97.

Yuan Z, Gobeil P, Campo M and Nasir L. (2010). Equine sarcoid fibroblasts over-express matrix metalloproteinases and are invasive. Virology 96: 143-51. 